\title{
Privacy Preservation of Sensitive Data using Polymorphic Encryption and Cryptographic Techniques
}

\author{
D.Asha Devi, M.Suresh Babu, K.Bhavana Raj
}

\begin{abstract}
The compilation and analysis of health records on a big data scale is becoming an essential approach to understand problematical diseases. In order to gain new insights it is important that researchers can cooperate: they will have to access each other's data and contribute to the data sets. In many cases, such health records involves privacy sensitive data about patients. Patients should be cautious to count on preservation of their privacy and on secure storage of their data. Polymorphic encryption and Pseudonymisation, form a narrative approach for the management of sensitive information, especially in health care. The conventional encryptionsystem is rather inflexible: once scrambled, just one key can be utilized to unscramble the information. This inflexibility is turning into an each more noteworthy issue with regards to huge information examination, where various gatherings who wish to research some portion of an encoded informational index all need the one key for decoding. Polymorphic encryption is another cryptographic strategy that tackles these issues. Together with the related procedure of polymorphic pseudonymisation new security and protection assurances can be given which are fundamental in zones, for example, (customized) wellbeing area, medicinal information accumulation by means of self-estimation applications, and all the more by and large in protection inviting character the board and information examination. Encryption, pseudonymization and anonymization are some of the importanttechniques that facilitate the usders on security of sensitive data, and ensure compliance both from an Data Regulation act and any other information security act like Health Insurance Portability and Accountability Act - (HIPAA) regulations.
\end{abstract}

Keywords- Encryption, Cryptographic technique, Anonymization, Pseudonymisation.

\section{INTRODUCTION}

The steps involved in Polymorphic encryption:

1. Legitimately after age, information can be scrambled in a 'polymorphic' way and put away at a (cloud) storeroom so that the capacity supplier can't get to. Vitally, there is no compelling reason to from the earlier fix that gets the chance to view the information, hence information can promptly be ensured. Eg : a PEP-empowered self-estimation gadget can accumulate its estimation information in polymorphically scrambled structure in a back-end information base.

2. Based on it tends to be chosen we can decode the information. This choice will be made based on an

Revised Manuscript Received on September 14, 2019.

D.Asha Devi, Professor, Department of ECE, Sreenidhi Institute of Science \& Technology, Hyderabad, Telangana, India. (E-mail ashadevi.d@rediff.com)

M.Suresh Babu, Professor, Department of CSE, K.L. University - off Campus - Hyderabad, Telangana, India. (E-mail: principaliis@ @rediff.com)

K.Bhavana Raj, Assistant Professor, KLHBS, K.L. University - off Campus - Hyderabad, Telangana, India. (E-mail: bhavana_raj_83@yahoo.com) arrangement, in which the information subject should assume a key job. The client of the Polymorphic encryptionempowered gadget can, for example, choose that specialists A, B. C may at some stage decode to utilize the information in their analysis, or therapeutic scientist bunches $\mathrm{X}, \mathrm{Y}, \mathrm{Z}$ may utilize for examinations, or outsiders $\mathrm{U}, \mathrm{V}, \mathrm{W}$ will utilize it for extra administrations, and so on.

3. 'tweaking' of scrambled information to make decryptable by a particular gathering should be possible in a visually impaired way. It should be finished by a confided in gathering who realizes how to change the ciphertext.

The innovation can give essential safetty and protection foundation for enormous information examination. The hidden numerical premise is shockingly straightforward for individuals with a sensible foundation in cryptography and yet shockingly amazing. Its capacity lies in the worldview that it gives, and in the new applications that it empowers. Consequently the estimation of the work lays less in the profundity of its cryptographic premise however in the expansiveness of the presentation situations. This will modify the manner in which we secure information in the time of huge information examination, with information originating from numerous bases.

A persuading go for improvement of PEP is to impel the safety and assurance invitingness of altered drug. The example in social protection changes fine-grained redid treatment techniques subject to verifiable out-happens to huge scale examination of patient data. In altered human administrations one needs to oversee simultaneously:_identifyable restorative information for analysis and healing of patients;

_ pseudonymised tolerant information for enormous scale restorative investigate;

_ the need to guarantee secrecy, respectability, legitimacy and accessibility of patient information;

- the capacity to deal with various wellsprings of patient information, incorporating into specific (wearable) selfestimation gadgets and applications.

The PEP structure is intended for this circumstance. It offers unprecented security insurance by means of encryption and pseudonymisation and simultaneously it bolsters the fundamental information get to usefulness for healing and investigation in customized medicinal services. Among the security objectives recorded in third projectile, the PEP framework focuses on privacy. In a far reaching 
way, different objectives should be ensured through different methods The PEP approach is pertinent in numerous different regions than social insurance. Be that as it may, this report focuses on wellbeing informatics: it utilizes outlines just from the medicinal services segment philosophy to different divisions, for example to deal with sensor or observation information in the internet of things.

The polymorphic encryption framework can be enhanced with a pseudonymisation foundation which is additionally polymorphic, and ensures that every being will naturally host various nom de plumes various gatherings and must be de-pseudonymised by members (like restorative specialists) who know the first character. It gives a prologue to Polymorphic Encryption and Pseudonymisation (PEP), at various degrees of reflection, concentrating on human services as application zone. Here it gives a vivid depiction of PEP, clarifying the fundamental usefulness for end user, enhanced by an explanation of the lawful structure given by the up and coming legislation of the European Union. The paper additionally contains a further developed, scientifically situated depiction of PEP, including the fundamental cryptographic natives, key and nom de plume, communication conventions, and so forth.

Numerous individuals these days utilize self-estimation gadgets and applications for monitoring their wellbeing and exercises, for example through watches that check steps, measure circulatory strain, or even take ECG's. These gadgets and applications touchy social or restorative information. The wellbeing information is considered as an uncommon class of information to which a larger amount of information security applies. Handling of unique classes of information is precluded, except if a special case applies.

Huge numbers of these applications and gadgets move the estimations to some focal database 'in the cloud' that is worked by the producer.

The information is then available for the client through uncommon applications or electronic records. Obligation of consideration applies. The exchange of information should just occur in encoded structure, as security against listening stealthily. Once moved, the information is in a perfect world put away in encoded structure as well, with the goal that a conceivable security occurrence does not quickly prompt loss of (plain, decoded) information. The gathering that has the decoding keys will approach the delicate information. These keys are expected to give clients access to their own information. Thus it is normally the producer who has the keys, and approaches all client information.

In present day information science, or (huge) information investigation, information is helpful for some reasons. Such adaptable utilization of the information is obstructed by encryption. In fact, customary encryption is consistently 'for a specific gathering', specifically for the gathering that has the unscrambling key. Nobody else can unscramble. The choice who can decode must be taken right now of encryption. In a various use situation, information are encoded, numerous gatherings must have the key.

fundamental advantage of polymorphic encryption is it permits increasingly adaptable utilization situations, where the decision who is permitted to decode can be delayed, and leaves it to the creative mind of the peruser to move the

while holding information insurance. This will be clarified pictorially in the following

\subsection{Traditional and polymorphic encryption, pictorially}

Encryption is a scientific strategy that makes information or ambiguous, however so that anybody possessing a particular 'cryptographic' key can make the encoded message decipherable once more. We will digest from the strategy for encryption and portraying it pictorially as putting a message in a chest with a lock. Just individuals with the suitable key can open the lock.

Hence, encryption of a message $M$ can be depicted as:

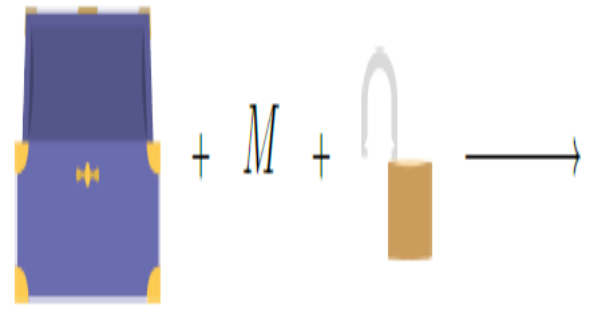

Encryption of message $M$

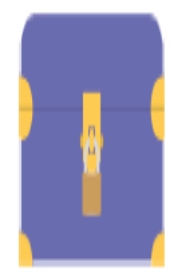

(1)
The locked chest willbe in store, where it will be sent to different place.

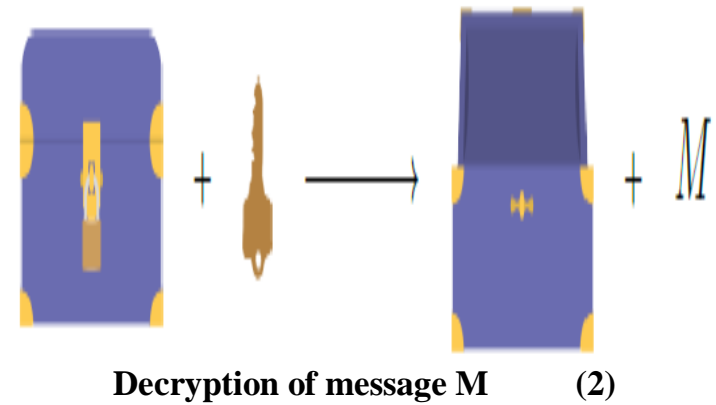

Here the chest first message $M$ flies out. This can be executed distinctly by somebody who has the single key that fits the lock. We consider the perfect circumstance where the correct key is totally vital, and the chest can't be openend in some other manner, for example by power. It might be conceivable however that various individuals have a duplicate of the single key that opens the lock.

By means of this chest similitude we can clarify some fundamental cryptographic wording. The message $M$ is known as the plaintext. In encoded structure, bolted inside the chest, it is known as the ciphertext. The open lock is known as an open key, and one that opens the lock is the related exclusive key.

When I'm the main individual that has such key, at that point I can circulate many open locks for this specific key freely accessible, with the goal that others can utilize it to encode message for me as in (1), which no one but I can unscramble, as in (2).

This is the substance of what is called 'open key' encryption.

A significant point is: when the lock is shut, there is just 
one key that can open it. in the event that various individuals need get to; they all need a duplicate of the key. We might want to have greater adaptability. Next we think about a comparative representation for polymorphic locks. We delineate this new idea as a lock with a wheel:
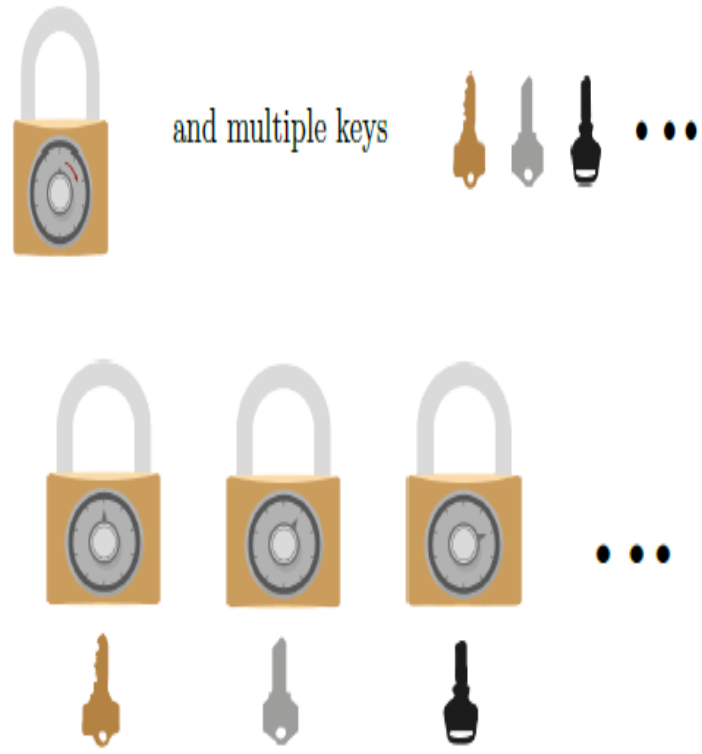

Here it will identify the various places of the wheel. In the event that it is 'up', just the dark colored key on the left opens the lock. However, on the off chance that the wheel is moved one score to one side, the dim key in the center fits, and no longer the darker one. Also, if the wheel is moved another indent, the dark key on the correct fits solely. The method permits a boundless number of such scores and subsequently a boundless number of relating keys for a solitary polymorphic lock. On a fundamental level, anybody can turn this wheel, however exceptional learning is expected to choose the correct wheel position, out of the numerous potential ones, with the goal that a specific key fits. We begin with a straightforward situation where touchy information from some self-estimation gadget, similar to a watch, should be put away safely in some distributed storage Facility.

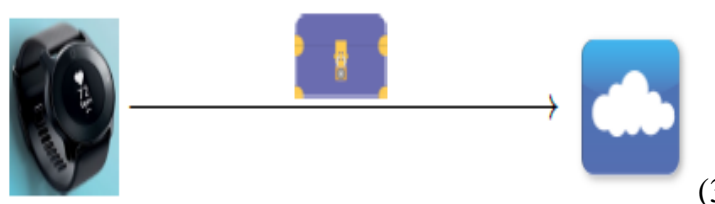

The cloud provider will not retrieve the data from the watch, as they are encrypted in a polymorphic manner.

The central converter only knows how to turn the wheel on a polymorphic lock so that keys of specific revelries suite.

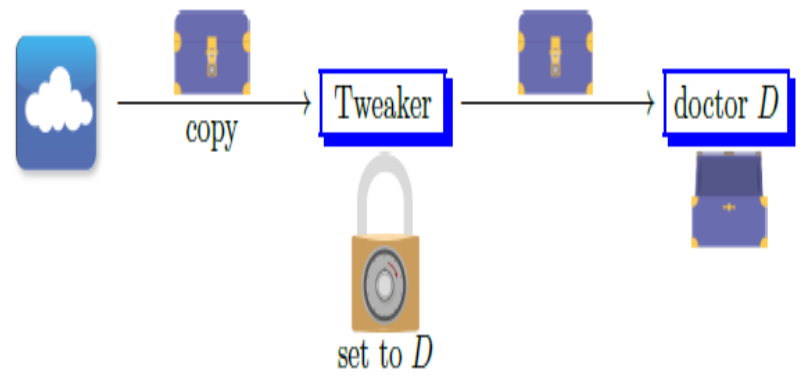

Polymorphic locks of specific parties (4)
The way toward turning the wheel on the lock will be called re-keying. In these charts (3) and (4) we see the intensity of polymorphic encryption: information is put away in scrambled structure, where the distributed storage supplier can't get to. Who gets access can be chosen later, by reasonably turning the wheel on the lock. In the representation it is specialist $\mathrm{D}$, however it can well be specialist $\mathrm{E}$ at some other stage, or a restorative scientist, or a specialist co-op.

There is this (trusted) transitional gathering, called the Tweaker, who realizes how to turn secures a particular way, with the goal that particular members can open the lock. Along these lines the Tweaker has a urgent, incredible position. In any case, the Tweaker works indiscriminately: it can't observe the information (the substance of the chest); it can just turn the wheel on a lock, outwardly of the chest. (Here we verifiably expect that the Tweaker isn't in control of any of the conceivable keys.)

At the point when an appropriate verification and access foundation is included, the client can set guidelines for the Tweaker and control utilization of the information. The client would then be able to make his own information accessible, for example for (open) logical research, however not for (private) business look into. Or then again he may control which individuals from the medicinal calling can't get to which information. On the off chance that this PEP approach forms into a standard, and 'Energy consistent' wearable's and applications become accessible, clients can be responsible for their information. The clever thought is that polymorphic encryption works in a conventional way, and the choices about who can unscramble need not be taken at the season of encryption. The scrambled ciphertext can be changed later, in a visually impaired way, with the goal that picked members can unscramble and gain admittance to the information.

\section{POLYMORPHIC PSEUDONYMISATION}

This approach comprises of both encryption and pseudonymisation, in polymorphic structure. This segment clarifies pseudonymisation, likewise through images with chests.

We need to take a gander at characters and nom de plumes. We accept that every member in the framework has a novel (individual) character, composed as pid. This is normally an extraordinary number, similar to a government managed savings number or some other (medicinal) enlistment number or identifier. We dynamic away from the subtleties: for member A we will compose pidA for an identifier that is interestingly connected with A. Such 'worldwide identifiers' are helpful for connecting information crosswise over various databases, yet they structure genuine protection dangers since they make it conceivable to break nearby settings and furthermore security chances for example as personality misrepresentation.

These pid's structure the reason for 'neighborhood' nom de plumes. Every member will host an alternate nom de 
plume various gatherings. For example, I will have various nom de plumes specialists $\mathrm{X}, \mathrm{Y}, \mathrm{Z}$, and at therapeutic research bunches $\mathrm{U}, \mathrm{V}, \mathrm{W}$.

The reason is as per the following. These gatherings could some way or another loses their information, or even malignantly consolidates information with others. On the off chance that various gatherings utilize various pen names a similar patient, it is on a fundamental level impractical to consolidate the information at any rate not based on identifiers. By and large, one talks about 'space explicit' nom de plumes; make it difficult to connect characters crosswise over various areas.

We will compose:

pidA@B for the pen name An at B.

Consequently, the various pen names tolerant $A n$ at specialists X; Y;Z are composed as pidA@X; pidA@Y; pidA@Z individually. They will now and again be called 'neighborhood' pen names, they are nearby to these various specialists. Specialists will accordingly store both the genuine name/character of their patients and their neighborhood nom de plumes.

Scientists will just have (their own) neighborhood nom de plumes, not personalities of patients.

The Tweaker assumes a focal job in framing these neighborhood nom de plumes, a visually impaired way.

Polymorphic lock

The different chests have an alternate shading: this utilize these red chests for nom de plumes, as in the past, the blue chests for information.

In any case, more significantly, these nom de plume have a wheel themselves: there isn't just a wheel on the lock, to make it polymorphic, yet additionally a wheel in favor of the chest, by the situation of the lock. By turning this subsequent wheel, the substance of it can be changed, in a visually impaired way, without opening the chest. We utilize this as pursues.

A is formed by putting A's personal identifier pidA locked in a red chest with a polymorphic lock:

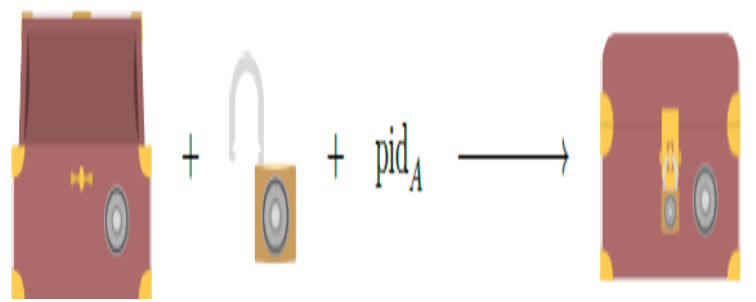

A's personal identifier pidA locked in a red chest with a polymorphic lock

The two main points are:

a nearby nom de plume would now be able to be developed inside the chest by turning the wheel on the chest to position B;

on the off chance that the wheel on the lock is additionally set in place $B$, at that point $B$ can open the bolted chest and locate the neighborhood assumed name pidA@B.

The subsequent box, with the two wheels appropriately turned, will be called an encoded nom de plume.

This set-up is progressively useful, as will be portrayed straightaway. We come back to the savvy situation from the

above area, and expand the convention with the uniqueness of the client.

Give An a chance to be the client/proprietor of the savvy. Expect that the watch by one way or another contains the character pidA of the proprietor, in a chest (as polymorphic nom de plume). At the point when the watch needs to offload information to a Storage Facility, it directs two chests to the Tweaker: The Tweaker does not contact the principal (blue) information chest. Be that as it may, it turns the two wheels on the second (red) personality chest, both to position SF, for the Storage Facility. Accordingly, the red chest contains the neighborhood pen name of client An at the Storage Facility. The Tweaker at that point passes the two chests on, for capacity: The Tweaker will not touch the initial data chest.

The Tweaker then passes both chests on, for storage:

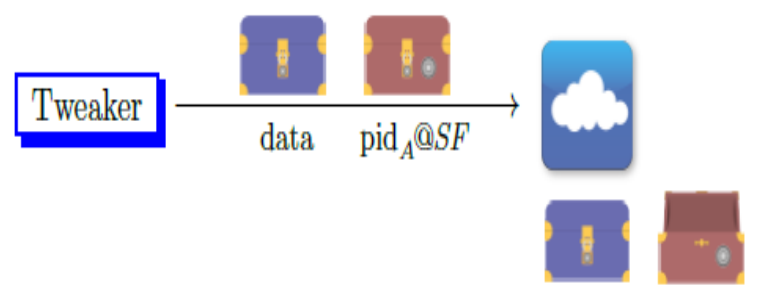

Tweaker passes both chests (7)

Since the wheel on the lock of the personality chest has additionally been gone to position SF, the Storage Facility can open this chest, with the goal that the nearby nom de plume flies out. SF utilizes this nom de plume a database key, where the blue information chest is put away; see

Figure 1.2 underneath :

A similar method is pursued whenever that the watch needs to off-load information. A similar nom de plume flies away from the Storage facility side, and the new blue chest is put away, under a similar database key, alongside the prior blue chest. Actually, a similar technique is likewise pursued when a therapeutic specialist has inspected An and needs to store the finding. The specialist or, the PC of the specialist places the finding information in a blue chest with a polymorphic lock, and the patient identifier pidA in a red chest and sends them two off to the Tweaker, as in (6). The Tweaker at that point continues as in (7), so the scrambled finding information is added to the database record with the watch information, under a similar database key. Figure 2 gives a sketch of such a record. Next we take a gander at a recovery situation. We accept that individual A visits a restorative specialist $B$, who needs to recover a few documents about A from the Storage Facility. At this stage we disregard the issue of document determination, and accept that it is by one way or another known how the proper record (in a chest) must be picked.

Specialist B knows the identifier pidA of An, and sends it off in a red character chest to the Storage Facility, by means of the Tweaker:

Doctor B knows the identifier pidA of A, and sends it off in a red identity chest to the Storage Facility, via the Tweaker: 


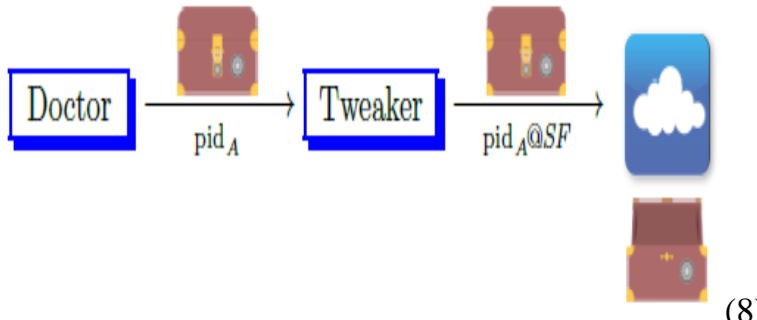

(8)

As sooner than, the Tweaker orchestrates the two wheels, on the chest and on the lock, to position SF, so that SF can open the chest and locate the neighborhood pen name. The Storage Facility at that point looks into the mentioned information, in a blue information chest, and returns the bolted chest by means of the Tweaker. The Tweaker alters the polymorphic lock with the goal that the key of the specialist fits, as in (4):

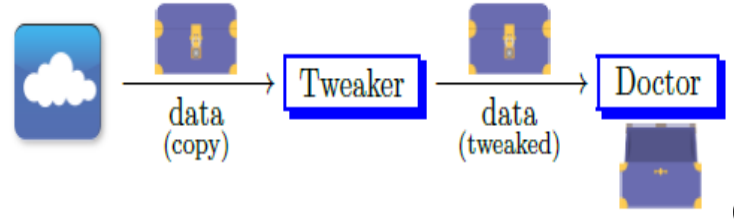

To outline, the PEP approach gives:

1. capacity of scrambled, pseudonymised information, with the goal that an intrigued, pernicious, or ineffectively secured SF will have slight impact on security threat;

2. consolidated capacity of information coming from a sources/gadgets;

3. retrievablility of the information for a particular individual, by an approved specialist or by the individual him/herself.

There is greater usefulness that we don't talk about :

A significant one is 'pseudonymous information sharing', where medicinal analysts can get to commonly after endorsement of their exploration plan by some oversight advisory group to pseudonymised however decoded information. It might happen that during therapeutic research, a gainful or disturbing sign is found in the restorative information of a specific individual, state A. In the event of such a 'unintentional finding' the nom de plume An at the exploration gathering can be made an interpretation of back to the neighborhood alias a medicinal specialist of $\mathrm{A}$, who can connect the nom de plume the genuine character, and educate A. Subsequently, depseudonymisation can just occur by gatherings who definitely know the first identity. An overview of the different parties and of the data owes between them is given in Figure 1.1.

\section{AUTHENTICATION, AUTHORISATION, AND SELECTION}

The above casual portrayal covers the center usefulness of the PEP approach. So as to form PEP into a useful framework with proper ensures greater usefulness should be included. Specifically, a framework must be included 'around' the Tweaker in Figure 1.1 for validation and authorisation of the different gatherings included, and furthermore for logging. This segment quickly talks about these issues at a conceptual level. similar individual however by means of various

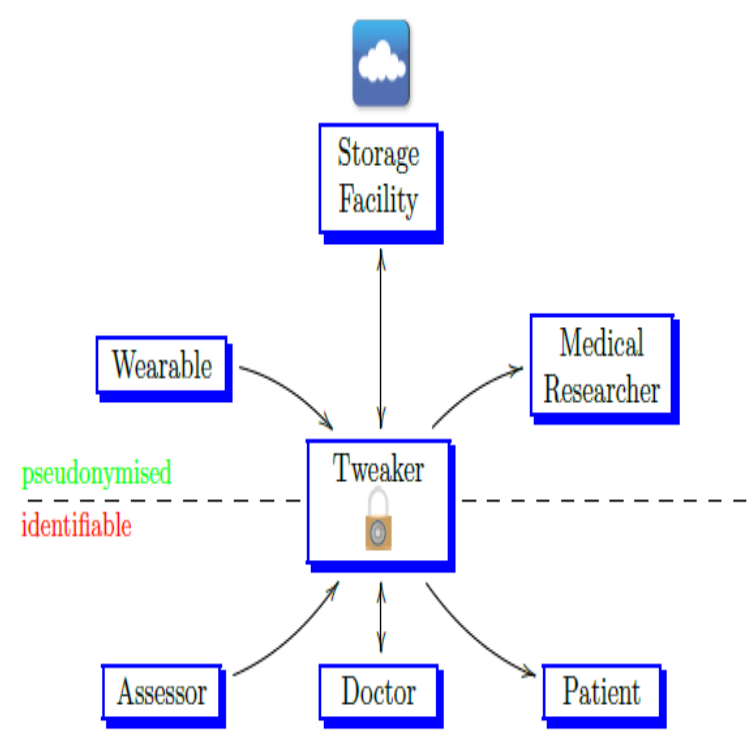

Figure 1.1: Interactions via the Tweaker.

At first we should say more regarding information stockpiling. So far we have referenced just that neighborhood aliases at the Storage Facility are utilized as database keys. The restorative substance of each record will be scrambled, through the blue information chests utilized above, yet some metadata should be included, with the goal that specialists, analysts or others can get to the proper information parts. An extremely straightforward picture of such a database passage is depicted in Figure 1.2, so as to pass on the thought. Accordingly, every blue chest that is put away, as in (6) and (7), must be joined by suitable metadata (names, dates, sources), with the goal that it very well may be put fittingly in this table. Essentially, every recovery demand in (8) ought to include a portrayal of the particular information that is mentioned.

The exact association of these database records isn't pertinent in the present setting. Rather than the somewhat subjectively picked names in the left segment in Figure 1.2, a standard restorative characterization framework ought to be utilized. The meta-information could likewise be cryptographically secured.

\begin{tabular}{c||c|c|l}
$\operatorname{pid}_{A} @ S F$ & date & source & content \\
\hline $\begin{array}{c}\text { Identity } \\
\text { insurance }\end{array}$ & $1 / 6 / 2003$ & doctor $X$ & \\
\hline ECG & $20 / 3 / 2016$ & watch & \\
\hline Pulse & $20 / 3 / 2016$ & watch & I \\
\hline Radiology & $15 / 2 / 2015$ & UMCRadboud & I \\
\hline$\vdots$ & $\vdots$ & $\vdots$ & $\vdots$
\end{tabular}

Fig 1.2: Neat diagram of a db record, with local pseudonym. 
with the goal that A can just observe his own documents. The (specialized) subtleties of this confirmation system are not pertinent here. It should give an adequate degree of assurance that pidA truly is An's own identifier, so it very well may be utilized to recover information, as in conventions, where the last beneficiary isn't 'Specialist', however A. This implies An ought to have his/her own private key and customer side programming to unscramble, that is, to open blue chests.

Moreover we predict that, after verification, client $\mathrm{A}$ gains admittance to a 'dashboard' that gives a review of, in addition to other things:

what information is put away about $\mathrm{A}$, that is, a posting of the record pidA@SF, as in Figure 1.2;

Log documents, depicting who has gotten to which information of An at which time;

The configurable arrangement of access rules, where client A can choose therapeutic state can gain admittance to which information; these principles may for example be founded on white posting, on boycotting, or on a mix;

A comparable arrangement of principles for other utilization of the information, together with reason portrayals. This 'other' use may incorporate, for example, business or non-business restorative research, or extra benefits, in light of a Data Licensing Agreement (DLA), see Section 1.5. On a basic level, the entire set up likewise permits that clients sell their information in pseudonymous structure, yet at the same time get the incomes independently. The exact association of such a dashboard includes numerous approach choices that are outside the extent of this paper. At long last, before proceeding onward to the cryptographic subtleties, we like to underscore the accompanying focuses.

1. PEP structure just focuses on cryptographic security of identifiers. Conceivable de-pseudonymisation (or 're recognizable proof') through the information is an entirely unexpected issue, which is significant, however out of extension. Such de-pseudonymisation may happen just in light of the fact that information contain identifiers which occurs for example every now and again with radiological pictures, where patient names are implanted or in light of the fact that blends of information lead to a profile that fits just one or a couple of individuals. There are numerous investigations about the renowned Netix and AOL cases demonstrating that re-distinguishing proof is frequently simpler than anticipated, particularly in blend with different databases or with open data for example from online networking.

2. In the PEP system the client isn't in finished power over his/her information. For example, a fake screen substance might be added to the image in Figure 1.1. On the off chance that specific conditions dictated by an enemy of extortion arrangement are met, the Tweaker can be requested to turn wheels on secures such a way, that the misrepresentation screen can decode. Such a set-up can be comprehended as an indirect access into the encryption. It might be defendable, or even attractive, in certain circumstances. All things considered we advocate maximal straightforwardness and responsibility.

\section{LEGAL STRUCTURE \& RESULTS}

The Polymorphic Encryption and Pseudonymisation structure tends to the issue of a person's command over his/her touchy individual information. The General Data Protection Regulation (GDPR) characterizes the accompanying information as touchy: 'individual information uncovering racial or ethnic starting point, political conclusions, religious or philosophical convictions, or worker's organization participation, and the preparing of hereditary information, biometric information with the end goal of exceptionally recognizing a characteristic individual, information concerning wellbeing. preparing of such information is denied as a matter of course; the fundamental exemption.

With regards to restorative research patients are frequently gone up against with purported 'cover' of 'expansive' educated assent structures. When investigating such shapes the assent isn't generally cover yet may undoubtedly be excessively wide. The reason determined in such assent structures is obviously medicinal research with regards to a specific infection or restorative field. On the off chance that all around clarified this structures a proper reason. The broadness lives in the incorporation of optional use for good purposes with respect to comparable restorative research, either over the span of a longitudinal report or for different examinations. The last effectively transforms the assent into a questionable assent on the off chance that one doesn't know about examples being utilized for completely various sorts of therapeutic issues.

The job of pseudonymisation in the GDPR.

Here it states that the utilization of pseudonymisation to individual information can decrease the dangers to the information subjects concerned and help controllers and processors to meet their information assurance commitments'. The general data protection characterizes pseudonymisation as 'the preparing of individual information so that the information can never again be ascribed to a particular information subject without the utilization of extra data, as long all things considered extra data is kept independently and subject to specialized and association measures to guarantee non-attribution to a recognized or recognizable individual'. We can infer that encryption is a type of pseudonymisation, regardless of whether the information controller can't get to the extra information (identifier); as long as somebody has a key dedistinguishing proof isn't irreversible and therewith the information are not viewed as unknown. Pseudonymisation is, notwithstanding, unequivocally qualified as information assurance as a matter of course, which alludes to architecting information minimization into the important specialized frameworks, and comparably qualified as what could be authored for instance of 'security by structure' . (a) GDPR Obviously the degree to which pseudonymisation 'considers' compelling information insurance will rely upon the potential for its inversion. 
The pseudonymisation that PEP empowers won't generally comprise pseudonymisation in the legitimate sense. This is because of the way that for this situation just the identifier is supplant by a pen name, the information may empower recognizable proof because of its linkability with other information (inside a similar database or subsequent to combining databases) or because of remarkable qualities that make conceivable the singling out of the person (which may likewise identify with the size of the information base). This implies those accessing information through the PEP system still have an obligation of consideration to guarantee the security of the information and clearly the authenticity of its handling. The dangers that preparing these information posture to the rights and opportunities of information subjects are, in any case, significantly diminished by pseudonymisation, which will most likely consider a type of Data Protection by Design. The job of Data Protection by Design and Default in European resolution.

Data Protection by Design (DPbD) must not be mistaken for Privacy by Design $(\mathrm{PbD})$, in spite of various connections and covers. The center differentiation is that while $\mathrm{PbD}$ might be a moral necessity, $\mathrm{DPbD}$ will before long be a lawful prerequisite. It is likewise essential to take note of that protection is an opportunity right, making it exceptionally difficult to characterize, not to mention structure or architect. $\mathrm{DPbD}$ requires incorporating information insurance with the specialized and authoritative design of individual information handling frameworks.

GDPR states: 'Considering the best in class, the expense of usage and the nature, degree, setting and reasons for handling just as the dangers of changing probability and seriousness for rights and opportunities of people and execute fitting specialized and authoritative measures, for example, pseudonymisation, which are intended to actualize information insurance standards, for example, information minimization, in a compelling way and to coordinate the fundamental shields into the handling so as to meet the prerequisites of this Regulation and ensure the privileges of information subjects.

GDPR: 'The controller will actualize suitable specialized and hierarchical measures for guaranteeing that, as a matter of course, just close to home information which are fundamental for every particular motivation behind the preparing are handled. That commitment applies to the measure of individual information gathered, the degree of their preparing, the time of their stockpiling and their availability. Specifically, such measures will guarantee that as a matter of course close to home information are not made open without the person's mediation to an uncertain number of characteristic people.

Since numerous substances engaged with enormous information investigation don't know about this (thinking there is just assent), these six grounds are summed up beneath.

a). the information subject has offered agree to the preparing of their own information for at least one explicit purposes;

b). handling is vital for the exhibition of an agreement to which the information subject is party or so as to make strides in line with the information subject before going into an agreement;

c). handling is essential for consistence with a lawful commitment to which the controller is subject;

d). handling is essential so as to ensure the crucial interests of the information subject or of another regular individual;

e). preparing is vital for the presentation of an errand completed in the open intrigue.

f). Preparing is vital for the motivations behind the genuine interests sought after by the controller or by an outsider. Point (f) of the first subparagraph will not have any significant bearing to handling did by open experts in the presentation of their undertakings'.

Kick can be founded on the principal ground, assent, which is generally joined with a protection arrangement or terms of administration. This guarantees information subjects host a reasonable outline of the gatherings that procedure their touchy information. The DLA can be short and far reaching, containing a progression of general conditions and a lot of measured statements some portion of which are discretionary. To guarantee that the information subject knows about every stipulation it can best be gotten to online to such an extent that every provision is appeared on a different screen. This gives individuals the alternative to rapidly navigate the whole DLA, and yet they are enticed to peruse every condition with consideration.

The DLA begins with recognizing the gatherings to the agreement: (1) the information subject: a patient or for example a client of a wellbeing App; and (2) the information controller(s): a recognized wellbeing App specialist organization, specialist, medicinal pro or for example an emergency clinic, insurance agency, examine establishment or Pharmaceutical Company. The upside of having a DLA rather than a unique assent or authorization framework is that the explanation and marking of the DLA makes familiarity with the immediate connection between the information subject and the gathering that desires to process his/her information as a component of enormous information investigation. This dodges bothersome system impacts of optional use by unidentified gatherings.

\section{CONCLUSION}

This paper privacy preservation of sensitive information utilizing Polymorphic encryption and cryptographic procedures gives the important security and protection foundation for enormous information investigation. The fundamental scientific premise is shockingly straightforward for individuals with a sensible foundation in cryptography and yet shockingly incredible. Its capacity lies in the new worldview that it gives, and in the new applications that it empowers. Subsequently the estimation of the work lies less in the profundity of various encryption techniques. This will change the manner in which we secure information in the period of enormous information investigation, with information originating from various sources. A spurring go for the improvement of PEP is to propel the safety and 
protection cordiality of customized medication. This new pattern in human services taking care of delicate information grows fine-grained customized treatment techniques dependent on measurable out-happens to enormous scale examination of patient information.

\section{REFERENCES}

1. Anor F.A. Dafa-Alla, EunHee Kim, Keun Ho Ryu, *Yong JunHeo "PRBAC: An Extended Role Based Access Control for Privacypreserving Data mining" In Proceedings of the Fourth Annual ACIS - IEE - 2006.

2. Alex Gurevich, Ehud Gudes "Privacy preserving Data MiningAlgorithms without the use of Secure Computation or Perturbation" IDAES

3. Murat Kantarcioglu, Chris Clifton. "Privacy preserving Distributed.Mining of association Rules on Horizontally partitioned Data.

4. Rakesh Agrawal, Srikant. Privacy Preserving Data Mining. ACM SIGMOD.

5. M. Naor and B. Pinkas, Oblivious Transfer and Polynomial Evaluation, Proceedings of the 31thAnnual Symposium on the Theory of Computing (STOC), ACM, 1999, pp. 245-254.

6. Anand Sharma and vibhaojha "“"Privacy preserving Data Mining by Cryptography" inSpringer-LNCS-CICSVol:89, "Recent Trends in Network Security and Applications" .pp.576- 581.

7. Jaideep Vaidya and Chris Clifton, "Leveraging the 'multi' in Secure Multiparty Computation,'WPES'03 October 30, 2003, Washington, DC, USA, ACM Transaction 2003, pp120-128.

8. T. ElGamal. A public key cryptosystem and a signature scheme based on discrete logarithms. IEEE Trans. on Information Theory, 31(4):469-472, 1985.

9. J. Kilian, Founding cryptography on oblivious transfer, ACM STOC '88, pp. 20-31.

10. M. Luby, Pseudorandomness and Cryptographic Applications, Princeton Computer Science 